\title{
Assessment of a vulnerability model against post-earthquake damage data: the case study of the historic city centre of L'Aquila in Italy
}

\author{
R. Ferlito ${ }^{1}$, M. Guarascio ${ }^{2}$ \& M. Zucconi ${ }^{3}$ \\ ${ }^{I}$ Italian Civil Protection Department, Rome, Italy \\ ${ }^{2}$ Department of Chemical Engineering Materials and Environment \\ (DICMA), University "Sapienza" of Rome, Italy \\ ${ }^{3}$ Department of Structural and Geotechnical Engineering (DISG), \\ University "Sapienza" of Rome, Italy
}

\begin{abstract}
The quantitative measure of seismic vulnerability is a necessary requirement for prevention and for an optimal emergency management.

Before L'Aquila's earthquake in 2009, the National Civil Protection, in collaboration with the University of L'Aquila, developed a model (Beolchini, "Definition of an indicator of urban vulnerability" (2003)) to evaluate the criticality seismic of urban centers by using a multidisciplinary approach.

The study has led to the definition of an urban vulnerability index, that allows to identify the most critical urban centers and the problematic elements, in order to establish a hierarchy for preventative measures and for an efficient emergency management (Calvi et al. "Development of seismic vulnerability assessment methodologies over the past 30 years" (2006).

One of the features of this evaluation is that it allows a simultaneous analysis of the multiple factors involved in risk assessment (Ferlito and Pizza "Modello di vulnerabilità di un centro urbano. Metodologia per la valutazione speditiva della vulnerabilità della viabilità d'emergenza" (2011); Ferlito et al. "Danger treblement de terre et mesure du risque à L'Aquila" (2010)).

After the seismic event occurred, same tests have been done to verify the validity of the model on the part of buildings, by comparing the criticality evaluated and damage suffered by the buildings, taking into account the local effects resulting from operations of microzonation. The seismic vulnerability
\end{abstract}


index is calculated as the weighted sum of same parameters, that represent a building feature which can affect the structural response under earthquake ground motion. Therefore, the influence of each parameter and their respective weights on the model have been analyzed. Sensitivity analyzes were performed to understand the model parameters that most affect structural vulnerability.

Furthermore the aim of this work is to provide a correlation between the indicator of criticality proposed here and the possible damage to the buildings resulting from an earthquake,

As for the test performed, the methodology has a good level of confidence in the modelling of the vulnerability of the buildings so it can be successfully used for seismic risk assessment, necessary for seismic safety and emergency management.

Keywords: vulnerability, seismic risk, building damage, prevention, emergency management.

\section{Introduction}

An urban settlement is to be considered as a functional system composed of various elements (for example: buildings, open spaces, infrastructure), each with a level of vulnerability that, when combined with the other, produce the overall vulnerability.

In this context it can be said that each function is guaranteed to the system at various levels (standards) and the damage is measurable as a loss of yield, so as decline of standards, and the vulnerability as a probability of a certain loss of performance in relation at a given seismic intensity.

A criterion for establish levels of critical standards can be used to distinguish the damage of a urban center in: an "elastic" phase (this does not preclude operations of subsystems, which are able to resume normal function independently); one of damage (partial system failure causes problems, but no collapse of subsystems, the urban system can guarantee a reduced functionality, but it includes all the essential functions for the emergency management) collapse (does not guarantee the essential functions for the management of the emergency, the aid of external structures is indispensable to overcome the most critical phase).

The process methodology to analyze the urban vulnerability can be the following:

1. Choosing a functional model, characterization of standards and critical levels of service

2. Identification of physical-mechanical structures (houses, schools, primary ways, systems, etc.), which are standards connected;

3. Vulnerability assessment of these structures;

4. Characterization of a relationship between level of structural damage and loss of system performance;

5. Determination of system vulnerabilities.

This policy setting has a potential utility for urban settings and effective policies. So, by identifying these elements (buildings, bridges, overpasses, 
tunnels, network systems, etc.), where earthquake damage is critical for functional decline or for the crisis of the system, there is a rational basis to proceed with a campaign of prevention seismic tendency to reconcile safety, cost, speed, reliability intervention.

For this purpose the urban system has been modeled as a superposition of multiple layers that contain the elements necessary to ensure the functions of the center. In particular the minimum functions that must be guaranteed after a seismic event have been identified. Other elements essential to the life of an urban center have been ignored, for example, commercial facilities, services, advanced tertiary, administrative and managerial activities, productive activities etc. The methodology will consider these factors when we switch to a more detailed definition of the problem.

In the present work, the attention has been focused on the layer of buildings. An indicator of vulnerability has been defined, whose calibration allows us to obtain its correlation with the level of predictable damage at a given intensity of the earthquake [5].

As with all components of the urban center, starting from the knowledge of the structural and functional layer buildings, we have identified elements that constitute it first, then the different types of buildings, which have been modeled through the combination of their characteristics.

From the analysis of the attributes we assessed vulnerability of the buildings, from which we can calculate the layer vulnerability for the entire area or for the single zone.

The attributes taken into account are considered the most relevant in determining the vulnerability of the element and are the most easily detectable by inspection expeditious. By a weighted sum of the attributes you get to vulnerability assessment of buildings.

The necessary data collection, essential for the elaborations, has been carried out on the basis of simplified techniques specifically identified.

\section{Calibration of the vulnerability model}

The methodology has been the object of various testing activities, especially since the earthquake of the $6^{\text {th }}$ April 2009, which involved the city of L'Aquila, which offered the opportunity to assess, in particular, the validity of the algorithm for the estimation of vulnerability of the buildings.

In fact it was possible to test the model of vulnerability proposed by comparing the assessment achieved with the post-earthquake results of fitness for human habitation and with the relief of damages [6].

To this aim we have used the most modern geomatic techniques [7, 8], using satellite images [9] taken a few days after the earthquake, that have allowed to identify the buildings that had suffered significant damage. A GIS platform has been realized: this comprises several layers, such as the vulnerability layer, the damage layer and the layer of fitness for human habitation, which can be put in relation rapidly and provides a synthetic and immediate result for the individual layers and for the combinations between the different layers. 
In an initial phase, comparisons were made by dividing the total perimeter in three homogeneous areas for extension and for the characteristics of the structural types, with a very good correspondence between the results of the outcomes of the fitness for human habitation and the vulnerability assessments, with positive tests in over $90 \%$ of cases, in the area not subject to seismic amplification.

To carry out a more rigorous analysis we have used a statistical linear regression model with dichotomous response that can be applied to a sufficiently large sample survey of empirical data. In particular, the regression was used for a sample survey carefully chosen between the buildings belonging to the historical city center, that are representative of the different building types present in the study area. The vulnerability assessments have been related to the fitness for human habitation, taking into account the local effects of microzonation associated with the area where are located the buildings.

Therefore the vulnerability and the amplification factor are the attributes associated with each building, and can express the probability of fitness for human habitation of the building in question, with a regression conditioning.

After have been taken into account the data collected with the form of damage detection, emergency intervention and fitness for human habitation for ordinary buildings in the post-seismic emergency - form AeDES - for the territory of Abruzzo. A sample of 21,835 masonry buildings has been analyzed, located in different areas, that are characterized by different values of the macroseismic intensity $\mathrm{I}_{\mathrm{EMS}}$.

The card AeDES is formed from several sections, including the identification of the building, the geometric and structural characterization, and the damage of the structural and non-structural components. From the form it is possible to derive the information necessary for the calibration of the vulnerability index.

\subsection{Vulnerability Index VI}

The parameters considered for the calculation of Vulnerability Index VI [10, 11], are contained in AeDES form. Therefore the sample survey of buildings relief in Abruzzo has been useful as an additional test of the model.

The data used are:

- prevalent type of structure;

- age of the building;

- presence of isolated columns;

- number of floors;

- type of roof;

- location of the building in the aggregate;

- presence of damage;

- site effects.

Index VI is evaluated by a weighted sum of the vulnerabilities assigned to each parameter using the following equation:

$$
V I_{j}=\sum_{i=1}^{n} v_{j, i} p_{i}
$$


where:

○ $\quad v_{j, i}$ is the representative value of the vulnerability assigned to the $i$-th parameter considered taking into account its characteristics and its influence on the behavior of the building $j$;

- $p_{i}$ is the weight that takes into account the importance of the parameter in the assessment of the susceptibility of the building.

These parameters were adjusted to obtain a correlation between the indicator of vulnerability and the effects of the earthquake in terms of fitness for human habitation. The evaluation of fitness for human habitation is a preliminary estimation. The main goal is to establish if the buildings struck by the earthquake can be used without risking human life. An identification of classes of increasing vulnerability has been obtained as a result of the activities of the relief damage of buildings. The result of the judgment of fitness for human habitation has to fall in one of the six possible categories specified in the chart:

A. Usable building;

B. Building temporarily unusable (everything or part): but usable after some intervention, which makes it usable in all of its parts, without danger for the residents;

C. Building temporarily unusable, to be investigated further: when building presents characteristics that make the evaluation uncertain;

D. Building partially unusable: the state of certain portions of the building could cause elevated risk for their occupants;

E. Building unusable due to structural, non-structural or geotechnical risks;

F. Building unusable due to serious external risk, in absence of consistent damage to the building: for example, an undamaged building contiguous to a building that could collapse.

For the analysis we have used a fitness of human habitation mean defined as the weighted sum of the results of the judgment of fitness for human habitation. Furthermore, as shown in the above formula to each parameter is assigned a weight representing its relative importance compared to other parameters.

\subsection{Calibration of the parameters of the vulnerability index}

To calibrate the parameters of the vulnerability index, it was first considered the prevalent structural typology: we identified 30 different types, characterized by different vertical and horizontal components, coincident with those provided in the tab AEDES, as shown in the table below. The values in the table show the total number of buildings used for each single type.

In addition to these, we examined many buildings formed by different structural types, but they have not been used for the calibration of the model parameters. For buildings characterized by different structural types was calculated, however, the Vulnerability Index, assuming the situation more difficult as possible, that the building was built entirely with the worst type of structure detected on the building itself. These assessments were useful in the next phase, in which we carried out the correlation between the VI and the damage. 
For each of the 30 structural types we evaluated outcomes of fitness for human habitation and realized their histograms. We paid particular attention to analyze how the variation of the horizontal structures affects the outcome of fitness for human habitation, considering the same vertical structure and vice versa.

The graph below shows the results of fitness for human habitation for the 30 structural types analyzed, by indicating the horizontal structures in numbers 1,2 , .., 6 and the vertical structures in letters A, B, ..,E (see table 1).

Table 1: $\quad$ Structural types analysed.

\begin{tabular}{|c|c|c|c|c|c|c|}
\hline & \multicolumn{5}{|c|}{ VERTICAL STRUCTURE } \\
\hline & & \multirow[t]{2}{*}{$\begin{array}{l}\text { Not } \\
\text { identified }\end{array}$} & \multicolumn{2}{|c|}{$\begin{array}{l}\text { Wall texture } \\
\text { uneven and of } \\
\text { poor quality }\end{array}$} & \multicolumn{2}{|c|}{$\begin{array}{l}\text { Wall texture } \\
\text { regular and of } \\
\text { poor quality }\end{array}$} \\
\hline & & & $\begin{array}{l}\text { No } \\
\text { chains }\end{array}$ & $\begin{array}{c}\text { With } \\
\text { chains }\end{array}$ & $\begin{array}{c}\text { No } \\
\text { chains }\end{array}$ & $\begin{array}{l}\text { With } \\
\text { chains }\end{array}$ \\
\hline $\begin{array}{l}\text { HORIZONTAL } \\
\text { STRUCTURES }\end{array}$ & & $\mathrm{A}$ & $\mathrm{B}$ & $\mathrm{C}$ & $\mathrm{D}$ & $\mathrm{E}$ \\
\hline Not identified & 1 & 456 & 405 & 67 & 131 & 42 \\
\hline Vault without chains & 2 & 6 & 725 & 96 & 98 & 15 \\
\hline Vault with chains & 3 & 0 & 38 & 74 & 13 & 28 \\
\hline Beams with deformable slab & 4 & 22 & 1352 & 268 & 587 & 176 \\
\hline Beams with semi-rigid slab & 5 & 23 & 1199 & 404 & 619 & 520 \\
\hline Beams with rigid slab & 6 & 32 & 297 & 387 & 670 & 3027 \\
\hline
\end{tabular}

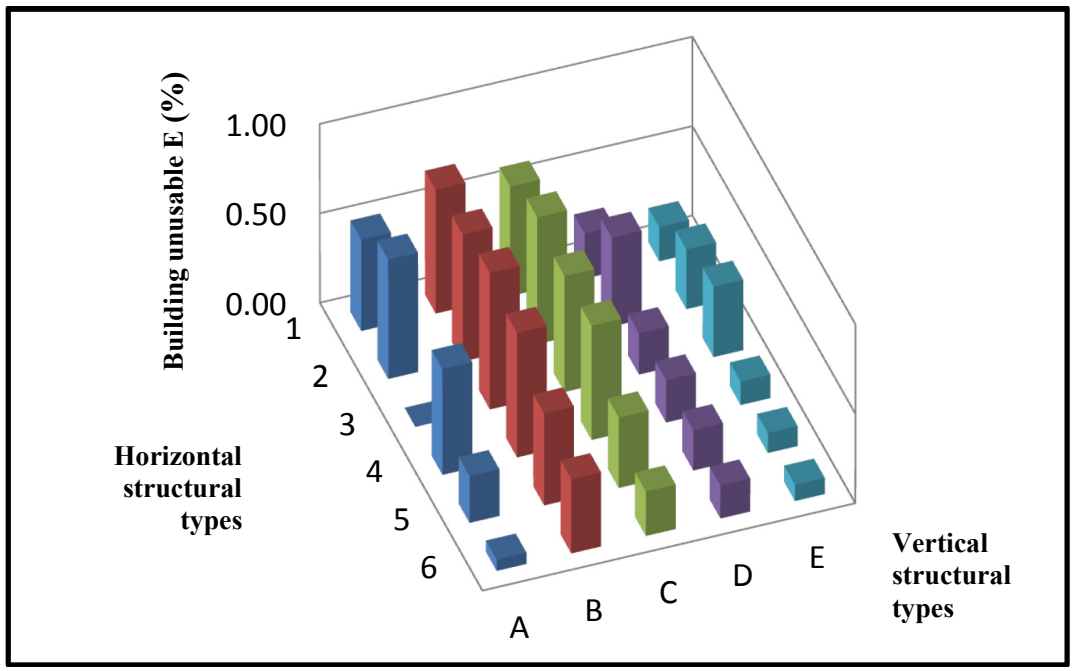

Figure 1: Unusable building $\mathrm{E}$ due to structural, non-structural or geotechnical risks depending on the structural type. 
Table 2 shows the percentages of unusable buildings E for the thirty structural types considered. Observing the values we can note that, for some types there are comparable percentages of unusable buildings. From the initial structural types considered, we identified four main classes, including structures similar to each other in terms of the structural response and therefore damage to the seismic action. Types can be identified in the table by 4 shades of gray.

Table 2: $\quad$ Percentage of unusable building E depending on the structural type.

\begin{tabular}{|c|c|c|c|c|c|}
\hline & $\mathbf{A}$ & B & C & D & $\mathbf{E}$ \\
\hline 1 & 0.51 & 0.69 & 0.61 & 0.25 & 0.19 \\
\hline 2 & 0.67 & 0.71 & 0.71 & 0.50 & 0.33 \\
\hline 3 & 0.00 & 0.76 & 0.65 & 0.23 & 0.39 \\
\hline 4 & 0.59 & 0.70 & 0.64 & 0.24 & 0.13 \\
\hline 5 & 0.26 & 0.51 & 0.39 & 0.22 & 0.11 \\
\hline 6 & 0.06 & 0.41 & 0.25 & 0.18 & 0.09 \\
\hline
\end{tabular}

The four types identified correspond to four classes of increasing vulnerability for which we have identified values to assign to the parameter structural type, as shown in the table below. Column E shows the percentage of buildings unusable for each type.

Table 3: $\quad$ Percentage of buildings unusable for each structural type.

\begin{tabular}{|c|c|c|}
\multicolumn{1}{c|}{} & E[\%] & $\boldsymbol{V}_{j i}$ \\
\hline TIP 1 & 0.66 & 4.5 \\
\cline { 1 - 1 } TIP 2 & 0.45 & 2.8 \\
\cline { 1 - 1 } TIP 3 & 0.24 & 1.0 \\
\cline { 1 - 1 } TIP 4 & 0.13 & 0.0 \\
\hline
\end{tabular}

The values to be assigned to the individual parameters have been calibrated in the range 4.5-0.0, where 4.5 indicates the maximum value of the class of vulnerability and 0.0 the minimum value. These values were obtained by scaling the expected value of building unusable medium (E) for each typological structural class in the range 4.5-0.0.

This interval was chosen as used in several models of vulnerability, including the GNDT second level approach from which different methodologies derived in the last decades, in order to make comparable the results obtained with our model.

Similarly we proceeded to the calculation of the other parameters that enter the evaluation of the vulnerability index in the range 4.5-0.0. 
In the tables below we reported the values assigned to each parameter.

For the parameter "age of the building" we took into account the age of the building construction as prepared in the form for the survey of vulnerability.

Table 4: Age of the building.

\begin{tabular}{|c|c|c|c|c|c|c|}
\hline Age of the building & $<1919$ & $19-45$ & $46-61$ & $62-83$ & $84-96$ & 1996 \\
\hline$V_{i}$ & 4.5 & 3.0 & 2.1 & 1.5 & 1.0 & 0.0 \\
\hline
\end{tabular}

As a further correction factor we took into account the presence of isolated columns, because as a result of the sample analyzed we found that in the presence of isolated columns there was a concentration of stress with an increase of localized damage. As shown in the following table, the value of the parameter is assigned in function of one of the four structural types considered.

Table 5: $\quad$ Presence of isolated columns.

\begin{tabular}{|c|c|c|c|c|}
\hline Isolated columns & TIP.1 & TIP.2 & TIP. 3 & TIP.4 \\
\hline $\mathbf{V}_{\mathbf{i}}$ & 4.5 & 2.6 & 1.3 & 0.0 \\
\hline
\end{tabular}

For the analyzed sample survey it is generally found that with the increase of the number of floors, there is an enhancement of the percentage of unusable buildings. Furthermore this percentage differs for building in aggregate or for alone building. For the moment it was decided to consider an average behavior, as it is already considered with the parameter "location of the building".

Table 6: Number of floors.

\begin{tabular}{|c|c|c|c|c|c|}
\hline Number of floors & $\mathbf{1}$ & $\mathbf{2}$ & $\mathbf{3}$ & $\mathbf{4}$ & $\mathbf{5}$ \\
\hline $\mathbf{V}_{\mathbf{i}}$ & 0.0 & 0.4 & 1.3 & 2.7 & 4.5 \\
\hline
\end{tabular}

It is also taking into account the type of building roof; in particular, a distinction is made between a pushing roof or not pushing roof and between a heavy or slight roof.

Table 7: $\quad$ Type of roof.

\begin{tabular}{|c|c|}
\hline Type of roof & $V_{i}$ \\
\hline Heavy pushing roof & 4.5 \\
\hline Slight pushing roof & 3.3 \\
\hline Not heavy pushing roof & 0.0 \\
\hline Not slight pushing roof & 1.2 \\
\hline
\end{tabular}

With this parameter, a distinction is made between a solitary building and a building site in aggregate, since their structural response to seismic action, especially in terms of damage was different.

Table 8: $\quad$ Location of the building in the aggregate.

\begin{tabular}{|c|c|c|c|c|}
\hline & \multicolumn{4}{|c|}{ Location of the building } \\
\hline & Corner building & Extremity building & Middle building & Alone building \\
\hline$V_{i}$ & 4.5 & 2.6 & 3.3 & 0.0 \\
\hline
\end{tabular}


Finally, the possible presence of fissures on the building and their extension was taken into account, using the European Macroseismic classification of damage EMS-98, which identifies 6 levels of damage: from no damage D0 to total collapse D5. Once you find the level of damage you have to evaluate the extension of the same, identifying if the damage affects less than $1 / 3$, between $1 / 3$ and $2 / 3$ or more than $2 / 3$ of the building surface.

Table 9: $\quad$ Presence of damage.

\begin{tabular}{|c|c|c|c|c|c|c|c|c|c|}
\hline \multirow[b]{2}{*}{ Damage extension } & \multicolumn{3}{|c|}{ D4-D5 } & \multicolumn{3}{|c|}{ D2-D3 } & \multicolumn{3}{|c|}{ D1 } \\
\hline & $>2 / 3$ & $1 / 3-2 / 3$ & $<1 / 3$ & $>2 / 3$ & $1 / 3-2 / 3$ & $<1 / 3$ & $>2 / 3$ & $1 / 3-2 / 3$ & $<1 / 3$ \\
\hline $\mathbf{V}_{\mathbf{i}}$ & & 4.5 & & 1 & 1.7 & & & 0 & \\
\hline
\end{tabular}

\section{Determination medium damage $D$ from Abruzzo data}

To determine the correlation between the indicator of vulnerability Iv and the damage, we referred to the average damage of individual buildings $\mathrm{D}$ normalized to 1 , calculated using the weighted combination of the damage observed on the different structural components considered by the board AEDES. The weights take into account various factors including the importance of the structural component in consideration on the behavior of the entire building and the extent of damage on the component itself.

Once rated the Medium Damage for each building we determined the expected value of the Medium Damage for the different outcomes of fitness for Human habitation, as shown in the following figure.

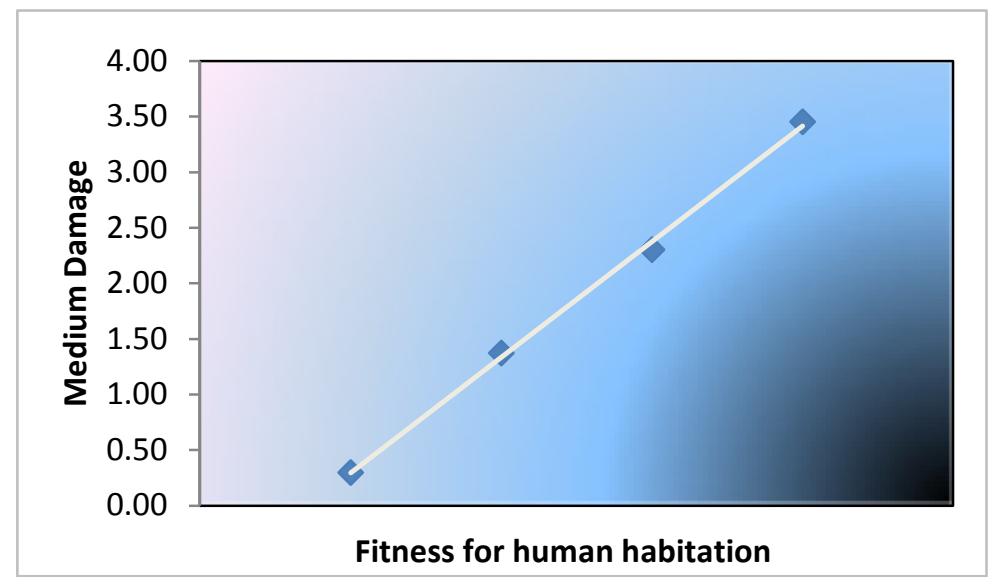

Figure 2: Expected value of the Medium Damage for the different results of Fitness for human habitation. 


\section{Correlation Iv-Dm}

Once defined the necessary parameters for the calculation of the Vulnerability Index VI, it was possible to calculate this index for the sample survey of masonry buildings in question. The index VI takes into account the characteristics of the building that affect their propensity to damage, so after considering the average damage calculated [12] by the combination of the damage of individual structural components, we evaluated the correlation between the two variables. For this purpose, for each intensity and for each level of average damage, the expected value of VI was assessed.

As an example we report below the results obtained for the case of macroseismic intensity 8.5 in tabular and graphic form.

Table 10: $\quad$ Expected value of Iv for each level of average damage.

\begin{tabular}{|c|c|c|c|}
\hline Damage & Number of buildings & Number of buildings [\%] & Average \\
\hline 0.5 & 217 & 0.21 & 0.33 \\
\hline 1 & 148 & 0.14 & 0.40 \\
\hline 1.5 & 2 & 0.00 & 0.67 \\
\hline 2 & 13 & 0.01 & 0.59 \\
\hline 2.5 & 99 & 0.10 & 0.57 \\
\hline 3 & 68 & 0.07 & 0.57 \\
\hline 3.5 & 53 & 0.05 & 0.65 \\
\hline 4 & 139 & 0.13 & 0.64 \\
\hline 4.5 & 126 & 0.12 & 0.65 \\
\hline 5 & 171 & 0.17 & 0.66 \\
\hline
\end{tabular}

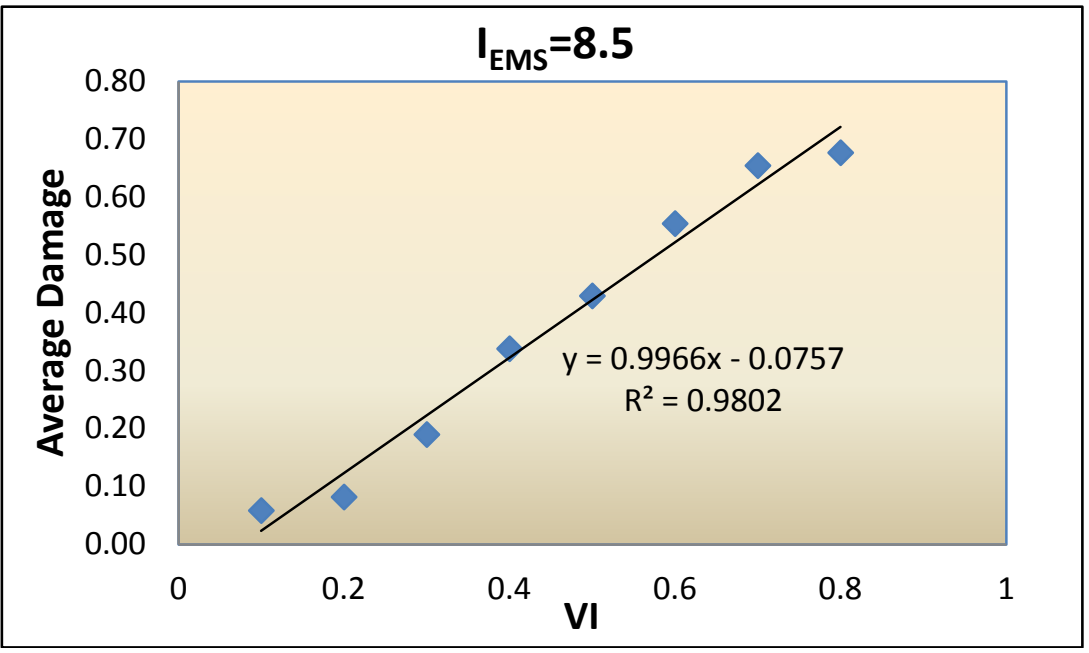

Figure 3: $\quad$ Expected value of VI for each level of average damage. 
From the graph above we can see that the relationship between the Vulnerability Index VI estimated and the average damage detected is coherent, indicating not only the validity of the modeling performed, but also the representativeness of the sample used sufficiently large to provide reliable results.

\section{Conclusions}

In conclusion, for every building in our data sample relative to the area of Abruzzo, affected by the April 6, 2009 earthquake, the Vulnerability Index VI has been calculated and appropriately calibrated, starting with the data derived from a survey of fitness for human habitation after the earthquake. Average damage was evaluated as well, starting with damage discovered to various structural components of single building.

Finally, for every homogeneous area in terms of macroseismic intensity, the correlation between the two abovementioned - VI and average damage - has been individualized, useful for future damage estimation starting with an expected survey of vulnerability.

The scope of this study, in fact, is to create a vulnerability model that permits the realization of appropriate scenario of damage, to utilize as support of preventive actions and for earthquake emergency plans other than to estimate economic and social loss.

\section{References}

[1] Giovanni Beolchini, "Definition of an indicator of urban vulnerability", Seismic Risk Conference and Urban Planning Scale: Analysis Methods and Tools of Intervention, Rome, 5-6 June 2003.

[2] G. M. Calvi, R. Pinho, G. Magenes, J.J. Bommer, L.F. Restrepo-Vélez and $\mathrm{H}$. Crowley. "Development of seismic vulnerability assessment methodologies over the past 30 years. ISET Journal of Earthquake Technology, Paper No. 472, Vol. 43, No. 3, September 2006, pp. 75-104.

[3] R. Ferlito, A. G. Pizza. "Modello di vulnerabilità di un centro urbano. Metodologia per la valutazione speditiva della vulnerabilità della viabilità d'emergenza". Ingegneria Sismica Anno XXVIII - N. 4 - ottobredicembre 2011.

[4] Ferlito R., Guarascio M., Zucconi M., Danger treblement de terre et mesure du risque à L'Aquila, École Centrale, no 578, July/August 2010.

[5] S. Giovinazzi, S. Lagomarsino. "Una metodologia per l'analisi di vulnerabilità sismica del Costruito” X Congresso Nazionale "L’ingegneria Sismica in Italia", Potenza-Matera 9-13 settembre 2001.

[6] A. Tertulliani, L. Arcoraci, M. Berardi, F. Bernardini, R. Camassi, C. Castellano, S. Del Mese, E. Ercolani, L. Graziani, I. Leschiutta, A. Rossi, M. Vecchi. "An application of EMS98 in a medium-sized city: The case of L'Aquila (Central Italy) after the April 6, 2009 Mw 6.3 earthquake”. May 2010. 
[7] Valerio Baiocchi, Donatella Dominici, Rachele Ferlito, Francesca Giannone, Massimo Guarascio, Maria Zucconi, (2011) Test of a building vulnerability model for L'Aquila earthquake. Applied Geomatics Springer.

[8] Valerio Baiocchi, Donatella Dominici, Rachele Ferlito, Francesca Giannone, Massimo Guarascio, Maria Zucconi, Analisi del rischio territoriale dovuto a sisma, 14a Conferenza Nazionale ASITA, 9 - 12 novembre 2010, Fiera di Brescia.

[9] Valerio Baiocchi, Filippo Del Guzzo, Donatella Dominici, Francesca Giannone, Maria Zucconi, Impiego di immagini satellitari EROS B per l'accertamento e la verifica dei danni post-sisma per la città dell'Aquila, 14a Conferenza Nazionale.

[10] D. Benedetti, V. Petrini. "Sulla vulnerabilità sismica di edifici in muratura: un metodo di valutazione".

[11] Romeu Vicente, Sonia Parodi, Sergio Lagomarsino, Humberto Varum, J. A. R. Mendes Silva. "Seismic vulnerability and risk assessment: case study of the historic city centre of Coimbra, Portugal". Bull Earthquake Eng (2011) 9:1067-1096 DOI 10.1007/s10518-010-9233-3.

[12] A. Bernardini, S. Giovinazzi, S. Lagomarsino, S. Parodi. "Vulnerabilità e previsione di danno a scala territoriale secondo una metodologia macrosismica coerente con la scala EMS-98." 